\title{
An advanced Jones calculus for the classification of periodic metamaterials
}

\author{
Christoph Menzel, ${ }^{1}$ Carsten Rockstuhl, ${ }^{1}$ and Falk Lederer ${ }^{1}$ \\ ${ }^{1}$ Institute of Condensed Matter Theory and Solid State Optics, \\ Friedrich-Schiller-Universität Jena, Max-Wien-Platz 1, D-07743 Jena, Germany
}

\begin{abstract}
By relying on an advanced Jones calculus we analyze the polarization properties of light upon propagation through metamaterial slabs in a comprehensive manner. Based on symmetry considerations, we show that all periodic metamaterials may be divided into five different classes only. It is shown that each class differently affects the polarization of the transmitted light and sustains different eigenmodes. We show how to deduce these five classes from symmetry considerations and provide a simple algorithm that can be applied to decide by measuring transmitted intensities to which class a given metamaterial is belonging to only.

PACS numbers: XX.XX.XX
\end{abstract}

\section{INTRODUCTION}

Metamaterials (MM) provide a large variety of unprecedented optical properties. Whereas in its infancy properties such as a dispersive permeability were at the focus of interest [1]3, the range of properties to be intentionally affected by suitably chosen MMs significantly increased. More and more complex [4-7] and most notably, chiral [8-14] or quasi-planar [15]17] chiral structures attracted a great deal of attention due to their polarization selective optical response and their potential to implement functional devices with unprecedented applications such as, e.g., broadband polarizers for circular light 18 . Moreover, from a scientific point of view, such complex MMs permit to observe unexpected and counterintuitive effects like asymmetric transmission for circular [19-22] or even for linearly polarized light 23 .

Recent studies have shown that the assignment of effective material parameters is doubtful in many cases [24] and generally requires the assumption of complex constitutive relations 25 27. Thus a more suitable target function to be tailored by an appropriate MM design is the optical response itself. This optical response is completely involved in the response functions, such as complex reflection and transmission coefficients, for a given input illumination. This paradigm change reflects that for an actual application a certain value of some effective material coefficient is of minor importance, as long as the sample exhibits the desired optical response.

The response, in particular in transmission, can then be easily described by transmittances and polarization ellipses, averaged polarization rotation and polarization conversion [8, 13, 28. These phenomenological quantities can be completely determined from the frequency dependent Jones matrix [29] that relates the complex amplitudes of the incident to the transmitted field. We will term this Jones matrix throughout the manuscript the T-matrix since it fully describes how the light is transmitted through a metamaterial slab. This $2 \times 2$ matrix comprises, in general, four different complex and dispersive quantities, reflecting the spectral properties of the MM. The associated Jones calculus can be applied to describe the transmission of an arbitrarily polarized incident plane wave through a MM slab if only the zeroth order Bloch mode emerges. This holds for MMs composed of periodically arranged sub-wavelength unit cells and we will assume this throughout the manuscript. For the sake of simplicity we also assume that the structures are symmetrically embedded. We assume that all materials are linear and reciprocal, i.e., excluding Faraday media. No further restrictions on the symmetry of the unit cells and the generally complex permittivity of the constituting materials are necessary.

With this work we intend to introduce a classification of periodic metamaterials based on their symmetry properties and to link them to their specific T-matrix. We will show that all metamaterials can be divided into only five distinct classes, each having an individual form of the Tmatrix and specific eigenstates. Each of these five classes leads to a very specific transmission characteristics directly linked to the symmetry of the structure. Therefore, this investigation provides a useful tool to analyze the optical response of complex MMs and it may serve as a guide to identify designs for a desired polarization response. Although for fabricated MM the geometry is usually known, the application of combinatorial approaches to explore new MM geometries in the near future requires such tool to classify the properties of MMs.

The paper is structured as follows. In Sec. II we present the necessary fundamentals to handle the generally complex valued T-matrices and derive general expressions for the eigenpolarizations. In Sec. III we derive the form of the T-matrix for the most relevant symmetry classes. In Sec. IV we provide examples of metamaterials for these symmetry classes and discuss briefly their optical behavior. In Sec. V a comprehensive tabular overview is given to summarize the results and we present a simple scheme to classify MM samples without having a priori knowledge in terms of the presented formalism by measured transmittances only. 


\section{BASIC THEORY}

It is assumed that the MM slab is illuminated by a plane wave propagating in positive $z$-direction

$$
\mathbf{E}_{i}(\mathbf{r}, t)=\left(\begin{array}{c}
I_{x} \\
I_{y}
\end{array}\right) e^{i(k z-\omega t)}
$$

with $\omega$ being its frequency, $k=\omega / c \sqrt{\epsilon(\omega)}$ the wavevector, and the complex amplitudes $I_{x}$ and $I_{y}$ describing the state of polarization. The transmitted field is then given by

$$
\mathbf{E}_{t}(\mathbf{r}, t)=\left(\begin{array}{c}
T_{x} \\
T_{y}
\end{array}\right) e^{i(k z-\omega t)},
$$

where we have assumed that the medium is sandwiched between a medium characterized by the permittivity $\epsilon(\omega)$. A sketch of the geometry is depicted in Fig. 1. The unit cells are periodically arranged in $x$ - and $y$-direction without restricting to a particular lattice. We assume coherent, monochromatic plane waves so to use a generalized Jones calculus instead of the Mueller calculus necessary for incoherent light 30, 31. The Jones calculus is said to be generalized since we allow for arbitrary complex Jones matrices which we will call T-matrices (transmission matrices).

The T-matrix connects the generally complex amplitudes of the incident and the transmitted field:

$$
\left(\begin{array}{l}
T_{x} \\
T_{y}
\end{array}\right)=\left(\begin{array}{ll}
T_{x x} & T_{x y} \\
T_{y x} & T_{y y}
\end{array}\right)\left(\begin{array}{l}
I_{x} \\
I_{y}
\end{array}\right)=\left(\begin{array}{ll}
A & B \\
C & D
\end{array}\right)\left(\begin{array}{l}
I_{x} \\
I_{y}
\end{array}\right)=\hat{T}^{\mathrm{f}}\left(\begin{array}{l}
I_{x} \\
I_{y}
\end{array}\right)
$$

where for convenience we have replaced the entries $T_{i j}$ by $A, B, C, D$ which form the actual T-matrix. In the following few subsections we will discuss some generic properties of this T-matrix.

\section{A. Directional dependent properties}

In the last term of Eq. (1) the T-matrix superscript $f$ designates propagation in forward direction. Of course, the choice of forward (f) and backward (b) propagation is arbitrary. Thus $\hat{T}^{\mathrm{b}}$ describes the transmission matrix for light propagating through the structure rotated by $180^{\circ}$ with respect to the $x$-axis, where the choice of $x$ or $y$ is arbitrary.

Since only reciprocal media are considered we have:

$$
\hat{T}^{b}=\left(\begin{array}{cc}
A & -C \\
-B & D
\end{array}\right)
$$

where the minus sign in the off-diagonal elements accounts for the rotation of the system looking from the backside [32. Therefore, the complex matrix $\hat{T}^{\mathrm{f}}$ already contains all information necessary to determine light transmission for arbitrarily polarized incident light from both main illumination directions. Its is important (a)

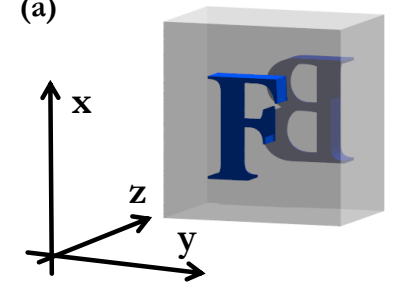

(b)

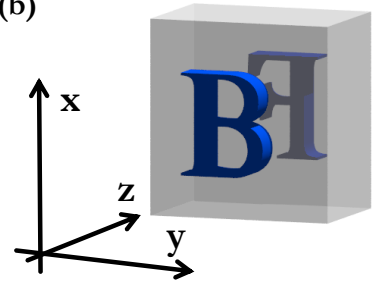

FIG. 1: Schematic of the geometry. a) and b) show the sample from opposite sides with $\mathrm{F}$ and $\mathrm{B}$ indicating the front- and back-side, respectively.

to stress that this relation between $\hat{T}^{\mathrm{f}}$ and $\hat{T}^{\mathrm{b}}$ is in general only valid for this particular base where the coordinate axis from the backside are given by replacing those of the frontside by $x^{\mathrm{b}}= \pm x^{\mathrm{f}}, y^{\mathrm{b}}=\mp y^{\mathrm{f}}$. The actual sign depends on the definition of the rotation of the system.

\section{B. Change of the base}

For analytical as well as for experimental concerns it is useful to have at hand the transmission matrix in an arbitrary not necessarily orthogonal base. Let the vectors $\mathbf{i}$ and $\mathbf{t}$ denote the incident and the transmitted light in a certain base. Then the incident and the transmitted light in the Cartesian base is given by $\mathbf{I}=\hat{\Lambda} \mathbf{i}$ and $\mathbf{T}=\hat{\Lambda} \mathbf{t}$, respectively, with $\Lambda$ being the change of basis matrix. Hence, the T-matrix for this new base is given by

$$
\mathbf{T}=\hat{T} \mathbf{I} \rightarrow \mathbf{t}=\hat{\Lambda}^{-1} \hat{T} \hat{\Lambda} \mathbf{i}=\hat{T}_{\text {new }} \mathbf{i}=\left(\begin{array}{ll}
T_{11} & T_{12} \\
T_{21} & T_{22}
\end{array}\right)\left(\begin{array}{l}
i_{1} \\
i_{2}
\end{array}\right) .
$$

All representations of the system are completely equivalent of course. A transformation of practical importance is the change from the Cartesian base to the circular base. Then the change of basis matrix reads as

$$
\hat{\Lambda}=\frac{1}{\sqrt{2}}\left(\begin{array}{cc}
1 & 1 \\
i & -i
\end{array}\right)
$$

where the columns of the $\hat{\Lambda}$ matrix are the new eigenstates. The T-matrix for circular states is then given by:

$$
\begin{aligned}
& \hat{T}_{\text {circ }}^{\mathrm{f}}=\left(\begin{array}{ll}
T_{++} & T_{+-} \\
T_{-+} & T_{--}
\end{array}\right)= \\
& =\frac{1}{2}\left(\begin{array}{ll}
{[A+D+i(B-C)]} & {[A-D-i(B+C)]} \\
{[A-D+i(B+C)]} & {[A+D-i(B-C)]}
\end{array}\right),
\end{aligned}
$$

connecting the amplitudes of circularly polarized incident and transmitted light:

$$
\left(\begin{array}{l}
T_{+} \\
T_{-}
\end{array}\right)=T_{\text {circ }}^{\mathrm{f}}\left(\begin{array}{l}
I_{+} \\
I_{-}
\end{array}\right),
$$


By using Eqs. (2) and (4) it becomes obvious that the T-matrix for backward propagation is given by:

$$
\hat{T}_{\text {circ }}^{\mathrm{b}}=\left(\begin{array}{cc}
T_{++} & -T_{-+} \\
-T_{+-} & T_{--}
\end{array}\right) .
$$

Note that the matrix $\hat{T}^{\mathrm{b}}$ in an arbitrary base is not simply given by Eq. (2), i.e. by interchanging the negative off-diagonal elements but by applying the corresponding change of basis matrix $\hat{\Lambda}$ to $\hat{T}^{\mathrm{f}}$ and $\hat{T}^{\mathrm{b}}$ in the linear base individually.

\section{Asymmetric Transmission}

Although not having discussed any symmetry property at all, we want to discuss at this point the special effect of asymmetric transmission which attracted considerable interest due to its counter-intuitive occurrence and discuss peculiarities related to a change of the base. The difference of the T-matrices for opposite propagation directions is the key to that asymmetric transmission. By asymmetric transmission $\Delta$ we understand the difference in the modulus of the total transmission between forward and backward propagation (see Fig. 1) for a certain base vector, e.g. $\mathbf{i}=i_{1} \mathbf{e}_{1}$ :

$$
\Delta=\left|T_{11}^{\mathrm{f}}\right|^{2}+\left|T_{12}^{\mathrm{f}}\right|^{2}-\left|T_{11}^{\mathrm{b}}\right|^{2}-\left|T_{12}^{\mathrm{b}}\right|^{2} .
$$

This quantity obviously depends on the chosen base, e.g., for a linear state coinciding with the coordinate axis we have $\left(\mathbf{i}=i_{x} \mathbf{e}_{x}\right)$ :

$$
\Delta^{\operatorname{lin}}=|B|^{2}-|C|^{2},
$$

whereas in the circular base we have $\left(\mathbf{i}=i_{+} \mathbf{e}_{+}\right)$:

$$
\Delta^{\text {circ }}=\left|T_{-+}\right|^{2}-\left|T_{+-}\right|^{2} \neq \Delta^{\text {lin }}
$$

in general. This dependency on the base is exploited e.g. in [19, 20] where asymmetric transmission for circularly polarized light is observed without asymmetric transmission for linear polarized light. Hence, the only proper choice is a linear base with base vectors parallel to the principal coordinate axes. Only in this base we can distinguish asymmetric transmission due to the structure from asymmetric transmission due to the chosen base.

\section{The Eigenpolarizations}

To characterize the different structures it is useful to determine the eigenstates of the polarization because they are uniquely related to the symmetry. Therefore, a simple eigenvalue problem has to be solved:

$$
\left(\begin{array}{ll}
A & B \\
C & D
\end{array}\right)\left(\begin{array}{l}
I_{x} \\
I_{y}
\end{array}\right)=\kappa\left(\begin{array}{l}
I_{x} \\
I_{y}
\end{array}\right)
$$

with the eigenvalue $\kappa$. By solving these equations we obtain:

$$
\kappa_{1,2}=\frac{1}{2}\left[(A+D) \pm \sqrt{(A-D)^{2}+4 B C}\right],
$$

where $\kappa_{1,2}$ gives the complex transmission for the eigenstates. The eigenpolarizations are then given by simply inserting $\kappa_{1,2}$ into Eq. (6) and solving for $I_{x}$ and $I_{y}$. The eigenbasis in matrix form can be written as

$$
\hat{\Lambda}=\left(\begin{array}{cc}
1 & 1 \\
\frac{\kappa_{1}-A}{B} & \frac{\kappa_{2}-A}{B}
\end{array}\right)
$$

with

$$
\mathbf{i}_{1}=\left(\begin{array}{c}
1 \\
\frac{\kappa_{1}-A}{B}
\end{array}\right) \text { and } \mathbf{i}_{2}=\left(\begin{array}{c}
1 \\
\frac{\kappa_{2}-A}{B}
\end{array}\right)
$$

where the eigenvectors are not normalized yet. It is important to note that the eigenbasis depends in general on the frequency due to the dispersive behavior of the transmission. Only for highly symmetric structures the eigenbasis is frequency independent as will be shown later. With the use of the characteristic polynomial of Eq. (6) the matrix $\hat{\Lambda}$ can be rewritten as

$$
\hat{\Lambda}=\left(\begin{array}{cc}
1 & \frac{\kappa_{2}-D}{C} \\
\frac{\kappa_{1}-A}{B} & 1
\end{array}\right)=\left(\begin{array}{cc}
1 & -\frac{X}{2 C} \\
\frac{X}{2 B} & 1
\end{array}\right),
$$

with $X=-(A-D)+\sqrt{(A-D)^{2}+4 B C}$. Note that the matrices $\hat{\Lambda}$ in Eqs. 8 and 10 are different but both are denoted simply by $\hat{\Lambda}$ not to confuse the reader with additional indices. They are only a concatenation of eigenvectors that are determined up to an arbitrary complex factor. The matrix $\hat{\Lambda}$ becomes unique as soon as the eigenvectors are normalized. The fractions in Eq. 10 are complex numbers, hence we can express the eigenbasis as:

$$
\hat{\Lambda}=\left(\begin{array}{cc}
1 & 1 \\
R_{1} e^{i \varphi_{1}} & \frac{1}{R_{2}} e^{-i \varphi_{2}}
\end{array}\right),
$$

with

$$
R_{1} e^{i \varphi_{1}}=\frac{X}{2 B} \text { and } R_{2} e^{i \varphi_{2}}=-\frac{X}{2 C} .
$$

The eigenvectors are obviously orthogonal only if

$$
R_{1}=R_{2} \text { and } \varphi_{1}+\varphi_{2}=(2 n+1) \pi \text { with } n \in \mathbb{Z} \text {. }
$$

This is only the case for linear, circular and a special class of elliptical polarization. In all other cases the eigenstates are non-orthogonal 33 35. Note that systems with orthogonal eigenstates are sometimes termed homogeneous systems whereas systems with non-orthogonal states are termed inhomogeneous ones [36].

Once the eigenstates are derived, the transmission matrix can be determined within this eigenbase by applying the transformation (3). The corresponding T-matrix 
is then diagonal. Nevertheless using the T-matrix in the eigenbase is only appropriate and convenient if the eigenstates are orthogonal and frequency independent.

The five different classes of periodic metamaterials that can be distinguished are closely related to their eigenstates. These five possible sets of eigenstates are linear, circular and elliptic ones, whereas the elliptic ones can be separated into co-rotating, counter-rotating and general elliptic states with no fixed relation between $\phi_{1}$ and $\phi_{2}$. Later on we will show how the symmetry class determines the respective eigenstate.

\section{SYMMETRY CONSIDERATIONS}

By the symmetry considerations in the next subsections we will show how the symmetry properties of the structure affect the symmetry of the T-matrix. The arising T-matrices can be reduced to five principal forms where in general a larger number of distinct matrices is possible by rotating the structure by an arbitrary angle with respect to the $z$-axis. On the other hand such rotations can be used to remove redundant information. Rotation by an angle $\varphi$ is accomplished by applying the following matrix operation:

$$
D_{\varphi}=\left(\begin{array}{cc}
\cos (\varphi) & \sin (\varphi) \\
-\sin (\varphi) & \cos (\varphi)
\end{array}\right) \rightarrow \hat{T}_{\text {new }}=D_{\varphi}^{-1} \hat{T} D_{\varphi}
$$

resulting in the new T-matrix $\hat{T}_{\text {new }}$ of the rotated sample. Note that the eigenvalues of the rotated system are invariant to this operation and are uniquely related to the principal symmetry. The actual form of the matrices and the derived, redundant matrices will be given later in Section IV to keep this part consistent.

In general all complex components of the T-matrix are different if the metamaterial does not exhibit any reflection or rotational symmetry. If such type of symmetry exists the components of the T-matrix must reflect that. We will therefore briefly discuss a varous symmetries and their corresponding impact on the T-matrices.

If the metamaterial is mirror-symmetric with respect to the $x-z$-plane, the T-matrix for the structure reflected at that plane is identical to the original one. Therefore we have:

$$
\begin{aligned}
& M_{x}=\left(\begin{array}{cc}
1 & 0 \\
0 & -1
\end{array}\right): M_{x}^{-1} \hat{T}^{\mathrm{f}} M_{x}=\left(\begin{array}{cc}
A & -B \\
-C & D
\end{array}\right)=\hat{T}^{\mathrm{f}} \\
& \rightarrow \hat{T}^{\mathrm{f}}=\left(\begin{array}{cc}
A & 0 \\
0 & D
\end{array}\right)
\end{aligned}
$$

with $M_{x}$ being the reflection matrix with respect to the $\mathrm{x}$-axis. So any structure that obeys that symmetry may be obviously described by a diagonal T-matrix.

If the metamaterial is mirror-symmetric with respect to the $y-z$-plane, we have:

$$
M_{y}=\left(\begin{array}{cc}
-1 & 0 \\
0 & 1
\end{array}\right): M_{y}^{-1} \hat{T}^{\mathrm{f}} M_{y}=\left(\begin{array}{cc}
A & -B \\
-C & D
\end{array}\right)=\hat{T}^{\mathrm{f}}
$$

$$
\rightarrow \hat{T}^{\mathrm{f}}=\left(\begin{array}{cc}
A & 0 \\
0 & D
\end{array}\right)
$$

Hence, if there exists any mirror plane parallel to the $z$-axis the T-matrix is diagonal provided that the mirror plane coincides with the $x$ - or $y$-axes, respectively. In such a system the eigenstates of the polarization are obviously linear states.

If the structure is $C_{2}$-symmetric with respect to the $z$-axis, we have:

$$
D_{\pi}=\left(\begin{array}{cc}
-1 & 0 \\
0 & -1
\end{array}\right): \quad D_{\pi}^{-1} \hat{T}^{\mathrm{f}} D_{\pi}=\left(\begin{array}{cc}
A & B \\
C & D
\end{array}\right) \equiv \hat{T}^{\mathrm{f}} .
$$

Hence rotating any structure by $180^{\circ}$ with respect to the $z$-axis does not change the response at all. Even if the structure does not have any further symmetry it fulfills that relation.

If the structure is $C_{3}$-symmetric with respect to the $z$-axis, we have:

$$
\rightarrow \hat{T}^{\mathrm{f}}=\left(\begin{array}{cc}
A & B \\
-B & A
\end{array}\right)
$$

However that symmetry is almost never met without additional metamaterial mirror symmetries but given here for completeness.

If the structure is $C_{4}$-symmetric with respect to the $z$-axis, we have:

$$
\begin{gathered}
D_{\frac{\pi}{2}}=\left(\begin{array}{cc}
-1 & 0 \\
0 & -1
\end{array}\right): D_{\frac{\pi}{2}}^{-1} \hat{T}^{\mathrm{f}} D_{\frac{\pi}{2}}=\left(\begin{array}{cc}
D & -C \\
-B & A
\end{array}\right)=\hat{T}^{\mathrm{f}} \\
\rightarrow \hat{T}^{\mathrm{f}}=\left(\begin{array}{cc}
A & B \\
-B & A
\end{array}\right) .
\end{gathered}
$$

Hence the structure is insensitive to linear polarized light of any state. If there is an additional mirror-symmetry with respect to a plane parallel or perpendicular to the $z$-axis, the off-diagonal elements will vanish resulting in an completely polarization independent structure. Otherwise the eigenstates will be circularly polarized as will be shown later in detail.

Further important conclusions can be drawn by investigating the possible mirror symmetries with respect to a plane perpendicular to the $z$-axis. If the structure possesses this type of symmetry, the reflected structure is the same as seen from the backside:

$$
\begin{aligned}
M_{x}^{-1} \hat{T}^{\mathrm{f}} M_{x}= & \left(\begin{array}{cc}
A & -B \\
-C & D
\end{array}\right)=\left(\begin{array}{cc}
A & -C \\
-B & D
\end{array}\right)=\hat{T}^{\mathrm{b}} \\
& \rightarrow \hat{T}^{\mathrm{f}}=\left(\begin{array}{cc}
A & B \\
B & D
\end{array}\right)
\end{aligned}
$$

i.e., the off-diagonal elements are identical. If the system possesses a center of inversion the matrix has also this 
form, because inversion is equivalent to applying a reflection and a subsequent rotation by $\pi$, where the latter does not change the response as shown in Eq. (16).

By comparison with Eq. $(18)$ it is obvious that the T-matrix will have the form $T^{\mathrm{f}}=\operatorname{diag}\{A, A\}$ if the structure is additionally $C_{4}$-symmetric with respect to the $z$-axis.

That important relation (Eq. (19)) is valid for all truly two-dimensional (planar) structures and all structures that possess any mirror plane perpendicular to a coordinate axis, i.e. achiral structures. In general, any substrate will break this symmetry [15, 16, 37, but usually the substrate effect is negligible compared to the effect of anisotropy [22].

Most important for our investigations are structures that cannot be mapped onto their mirror image by proper rotations. Those structures are called chiral. In general, the components of the T-matrix for those structures are all different. In the context of the basic geometry analyzed here there exist only two exceptions. The first one is already discussed within the context of Eq. (18). The second one is an $C_{2}$-symmetry with respect to the $x-$ or $y$-axis. For this type of symmetry the structure is identical from both sides, hence

$$
\hat{T}^{\mathrm{f}}=\hat{T}^{\mathrm{b}}=\left(\begin{array}{cc}
A & B \\
-B & D
\end{array}\right) .
$$

\section{EXAMPLES AND CLASSIFICATION}

To understand the usefulness of the approach presented, we will discuss the different symmetry classes for simple examples. The metaatoms exemplarily shown in the following are assumed to be periodically arranged in $x$ - and $y$-direction. Importantly, the symmetry constraints applied to the unit cell have to be consistent with the symmetry of the lattice. That is crucial since e.g. even an achiral metaatom, can result in a chiral structure by a proper arrangement on a periodic lattice [38.

\section{A. Simple anisotropic media}

The most significant symmetry is that of reflection symmetry with respect to the $x$ - or $y$-axis or both. As already explained within the context of Eqs. (14) and (15), thes T-matrix is then diagonal. The eigenvalues are simply $\kappa_{1}=A$ and $\kappa_{2}=D$. The eigenstates are linear states parallel and orthogonal to the mirror plane, respectively. Only a dichroitic behavior will be obtained and no polarization rotation occurs for light being parallel or orthogonal to the mirror planes. If the coordinate system is not aligned parallel to the mirror plane, the T-matrix for that system will have off-diagonal elements, which disappear after a proper rotation. The most general form of (a)

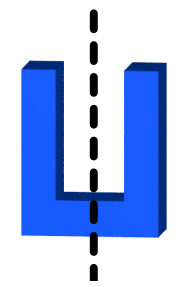

(b)

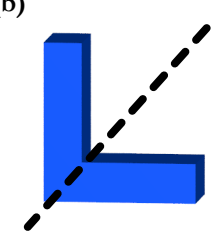

(c)

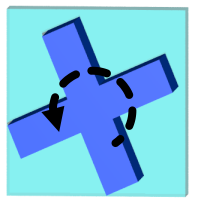

FIG. 2: Examples for simple anisotropic $(\mathrm{a}, \mathrm{b})$ and simple chiral (c) metaatoms. The structures are located in the $x-y$ plane with light impinging normally to the structure in $z$ direction. The black dashed lines indicate the mirror planes and the rotation axis respectively. (a) Split ring resonator with mirror plane parallel to the $y$-axis. (b) L-shaped particle with identical arms with mirror plane $45^{\circ}$ inclined. (c) Cross on substrate with $C_{4}$ rotational symmetry with respect to the $z$-axis. The square shaped substrate indicates the arrangement on a square lattice, necessary for the $C_{4}$ of the entire system. Such an arrangement gives rise to so-called structural chirality although the particle itself is achiral.

the T-matrix for systems with linear eigenstates is

$$
\hat{T}^{\mathrm{f}}=\left(\begin{array}{ll}
A & B \\
B & D
\end{array}\right)
$$

but in this case the components $A, B$ and $D$ are not independent but connected by trigonometric functions as is clear by explicitly evaluating Eq. (13) for a diagonal matrix.

An example for such a metamaterial is shown in Fig. 2 (a). Other examples are the fishnet [39] and its variations [40], cut wire pairs [41] and similar structures. In Fig. 2 (b) we have shown a special example of a structure with a symmetry plane which is $45^{\circ}$ inclined with respect to both the $x$ - and $y$-axis. In this case the Tmatrix has the form:

$$
\hat{T}^{\mathrm{f}}=\left(\begin{array}{ll}
A & B \\
B & A
\end{array}\right) .
$$

The eigenstates are linearly orthogonal polarized, hence a rotation by an angle $\varphi=45^{\circ}$ leads to a diagonal form:

$$
\hat{T}_{\text {new }}^{\mathrm{f}}=D_{\frac{\pi}{4}}^{-1} \hat{T}^{\mathrm{f}} D_{\frac{\pi}{4}}=\left(\begin{array}{cc}
A^{\prime} & 0 \\
0 & D^{\prime}
\end{array}\right)=\left(\begin{array}{cc}
A+B & 0 \\
0 & A-B
\end{array}\right) .
$$

A similar structure obeying the same relations is that published in [6]. There, the unit cell consisting of four split-ring resonators has no rotational symmetry. But reflecting the structure at a plane diagonal to the given unit cell leads to a structure that is shifted by half a period in $x$ - or $y$-direction. Due to the invariance of the optical response for periodic systems to any translation, this mirror plane leads in fact to linearly polarized eigenstates. Therefore, rotating the structure by $45^{\circ}$ results in a diagonal T-matrix. 


\section{B. Simple chiral media}

The second important group are those structures exhibiting $C_{4}$-symmetry but without any additional reflection symmetry. The T-matrix is then given by Eq. (18). Since these matrices are invariant to an arbitrary rotation $D_{\varphi}$, Eq. 18$]$ is already the most general form of the T-matrix in a linear orthogonal base for such systems. The T-matrix in the circular base is then diagonal:

$$
T_{\text {circ }}^{\mathrm{f}}=\left(\begin{array}{cc}
T_{++} & 0 \\
0 & T_{--}
\end{array}\right)=\left(\begin{array}{cc}
A+i B & 0 \\
0 & A-i B
\end{array}\right) .
$$

Obviously the eigenpolarizations are circular states, since the T-matrix is diagonal and the eigenvalues are simply $\kappa_{1}=A+i B$ and $\kappa_{2}=A-i B$. The $y$-components of the eigenvectors (Eq. (9) ) are $I_{y, 1,2}= \pm i$, i.e. frequency independent. At such systems all effects related to circular dichroism are observable whereas emphasis is put on the fact that circular dichroism is in general accompanied by a difference in the phase advance for right- (rcp) and left-circular polarized (lcp) light due to causality, i.e., the real and imaginary part of the wavenumber for rcp and lcp differ in general [42].

The difference $T_{++}-T_{--}=2 i B$ is given by the off-diagonal elements in the linear polarization representation and specifies the optical rotation power. Systems obeying that symmetry are prototypical optically active materials. Examples are gammadions, swastikas (see Fig. 2(c)) or $C_{4}$-spirals. Note that the influence of the substrate is important for planar structures [16, 37] the rotation power of which is independent of the structure height since it is a result of the substrate only.

\section{Generalized anisotropic media}

The third group consists of those systems that have a mirror symmetry perpendicular to the $z$-axis or a center of inversion and at most a $C_{2}$-symmetry with respect to the $z$-axis. From the latter one we know, that it has no influence on the transmission matrix (Eq. (16)). Examples are given in Fig. 3. The only necessary symmetry is the reflection symmetry perpendicular to the $z$-axis without any further restrictions. Hence, there is no preferable alignment in the $x-y$-plane and the basic form of the T-matrix is unaffected by any rotation with respect to the $z$-axis.

For those systems the T-matrices in the linear and circular representation are given by:

$T^{\mathrm{f}}=\left(\begin{array}{ll}A & B \\ B & D\end{array}\right), T_{\text {circ }}^{\mathrm{f}}=\frac{1}{2}\left(\begin{array}{cc}A+D & A-D+2 i B \\ A-D-2 i B & A+D\end{array}\right)$,

hence the eigenstates are neither linearly nor circularly states polarized.

Since we have $T_{++}=T_{--}$there is no polarization rotation due to chirality. In fact, it can be shown that the averaged polarization rotation accounting for chirality vanishes in such systems 43. The off-diagonal elements in the circular basis are different, hence the polarization conversion from left- to right-hand polarized light and vice versa is different. The difference in
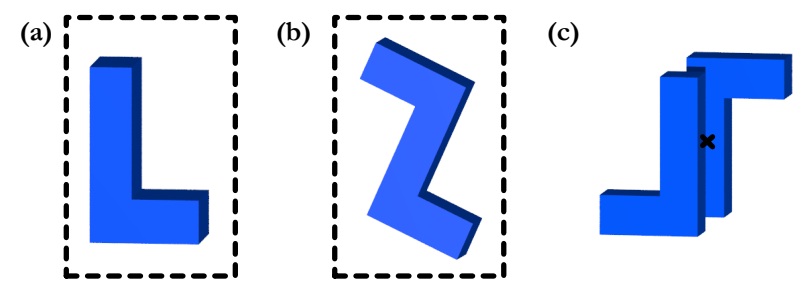

FIG. 3: Examples for generalized anisotropic metaatoms. The metaatoms are located in the $x-y$ plane with light impinging normally to the structure in $z$-direction. The black dashed lines indicate the mirror planes $(\mathrm{a}, \mathrm{b})$ and the black cross (c) a center of inversion symmetry, respectively. (a) A planar Lshaped metaatom with different arms. (b) A planar S-shaped metaatom with a $C_{2}$-symmetry with respect to the z-axis. (c) Three-dimensional metaatom made of L-shaped particles with a center of inversion.

conversion is again given by the off-diagonal elements in the linear basis $T_{+-}-T_{-+}=2 i B$. This difference is also the source of the asymmetric transmission for circularly polarized light. Assuming (+)-polarized incident light the total transmission $\tau$ in forward direction is $\tau^{\mathrm{f}}=\left|T_{++}\right|^{2}+\left|T_{-+}\right|^{2}$, whereas for the backward direction we have $\tau^{\mathrm{b}}=\left|T_{++}\right|^{2}+\left|T_{+-}\right|^{2}$ due to Eq. 19. Therefore, the difference in the total transmission is determined by $B$. For (-)-polarized incident light, the results are identical. Note that there is no asymmetric transmission for linear polarized light, as $\hat{T}^{\mathrm{f}}$ is symmetric.

It is important to note that the moduli of the offdiagonal elements are in general in the order of those of the diagonal elements $\left(10^{-1}\right)$. Hence the asymmetric transmission can become quite large. As already indicated before, any substrate will break the mirror symmetry in $z$-direction resulting in $B \neq C,|B-C| \ll$ $|B|, B \approx C$. As this difference due to the small effect of the substrate is very weak (typically $10^{-3}$ ), it is often neglected and hardly measurable compared to the asymmetric transmission effect.

The eigenstates for such a system are elliptical, corotating states, as discussed e.g. in [44. The effects of light propagating through such structures can be understood in terms of the concept of elliptical dichroism [45]. By using Eqs. 11, 12 and $B=C$ they can be expressed in normalized form as

$$
\mathbf{i}_{1}=\frac{1}{\sqrt{1+R^{2}}}\left(\begin{array}{c}
1 \\
R e^{i \varphi}
\end{array}\right), \mathbf{i}_{2}=\frac{R}{\sqrt{1+R^{2}}}\left(\begin{array}{c}
1 \\
-\frac{1}{R} e^{-i \varphi}
\end{array}\right) .
$$

They are only orthogonal for $\varphi=n \pi$ with $n \in \mathbb{N}$ leading to linear eigenstates.

Note that planar structures with that symmetry can be described by an effective permittivity tensor indepen- 
dent on the wavevector, i.e. without magnetoelectric coupling [46]. That is why we call this group generalized anisotropic structures.

The most general form is again obtained by applying a rotation by an arbitrary angle $\varphi$ leading to

$$
\hat{T}_{\text {new }}^{\mathrm{f}}=D_{\varphi}^{-1} \hat{T}^{\mathrm{f}} D_{\varphi}=\left(\begin{array}{cc}
A^{\prime} & B^{\prime} \\
B^{\prime} & D^{\prime}
\end{array}\right),
$$

hence the general form is invariant since no preferred alignment exists.

\section{Generalized chiral media}

The forth group are chiral structures that have an additional $C_{2}$-symmetry with respect to the $x-$ or $y$-axis. The T-matrix obeys the form:

$T^{\mathrm{f}}=\left(\begin{array}{cc}A & B \\ -B & D\end{array}\right), T_{\text {circ }}^{\mathrm{f}}=\frac{1}{2}\left(\begin{array}{cc}A+D+2 i B & A-D \\ A-D & A+D-2 i B\end{array}\right)$,

hence there is no difference in the polarization conversion and hence no asymmetric transmission neither for linear nor for circular polarized light. Furthermore there is obviously no asymmetric transmission in any base, since the structure is identical from both sides when the axis of rotation coincides with the $x$ - or $y$-axis.

But there is a difference in the quantity $T_{++}-T_{--}=$ $2 i B$ determining the optical rotation power typical for (a)

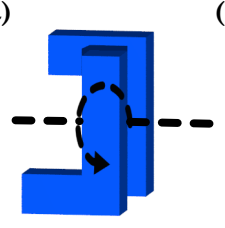

(b)

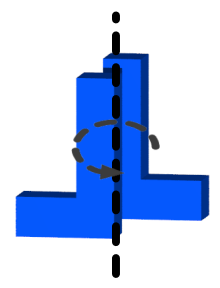

(c)

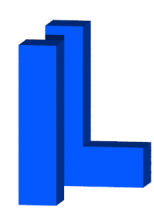

FIG. 4: Examples for generalized chiral metaatoms (a,b) and a no-symmetry metaatom (c). The structures are located in the $x-y$ plane with light impinging normally to the structure in $z$-direction. The black dashed lines indicate the axes of rotational symmetry $(\mathrm{a}, \mathrm{b})$. (a,b) Three-dimensional structures made of two L-shaped particles with $C_{2}$-symmetry with respect to the $x$ - or $y$-axis, respectively. They are identical for forward and backward propagation. (c) A three-dimensional structure made of an L-shaped particle and an I-shaped particle with no symmetry at all.

chiral structures. In contrast to the second group we have an additional anisotropy $(A \neq D)$ hence the eigenstates are not circular but elliptical counter-rotating. Again, by using Eqs. 111), 12 and $C=-B$ they can be expressed in normalized form as

$$
\mathbf{i}_{1}=\frac{1}{\sqrt{1+R^{2}}}\left(\begin{array}{c}
1 \\
R e^{i \varphi}
\end{array}\right), \mathbf{i}_{2}=\frac{R}{\sqrt{1+R^{2}}}\left(\begin{array}{c}
1 \\
\frac{1}{R} e^{-i \varphi}
\end{array}\right) .
$$

They are only orthogonal if $\varphi=\frac{\pi}{2}+n \pi$ with $n \in \mathbb{N}$ leading to circular counter-propagating eigenstates typical for chiral structures. That is why we term this group generalized chiral structures.

Typical examples are shown in Fig. 4 a) and b). Another important example are three-dimensional spirals [47 51] with $\frac{N}{2}$ whorls aligned along the $z$-axis. Spirals with integer whorls are clearly identical for both propagation directions, whereas spirals with half-integer whorls are identical after rotation by $\pi$ around the $z$-axis keeping the response unaffected.

Note that for an arbitrary rotation $D_{\varphi}$ all matrix elements are different, hence the symmetry axis must be aligned with a principal coordinate axis to achieve the form of Eq. (21). In particular if the system is rotated by $45^{\circ}$ the T-matrix has the form:

$$
T^{\mathrm{f}}=\left(\begin{array}{ll}
A^{\prime} & B^{\prime} \\
C^{\prime} & A^{\prime}
\end{array}\right)
$$

Nevertheless if the eigenvectors of the arbitrarily oriented system are elliptical counter-rotating the convenient form of Eq. (21) can be achieved by a proper alignment of the system.

\section{E. Arbitrary complex media}

The fifth and last group are chiral structures without any symmetry. A simple example is shown in Fig. 4 c). Here all elements of the T-matrices in the linear as well as in the circular base are different:

$$
T^{\mathrm{f}}=\left(\begin{array}{ll}
A & B \\
C & D
\end{array}\right) .
$$

It is impossible to achieve $|B|=|C|$ by a proper rotation. Therefore, independent of the base asymmetric transmission occurs always and in particular also for linearly polarized light. All effects of generalized anisotropy as well as generalized chirality can be observed. The normalized eigenvectors can be expressed as:

$$
\mathbf{i}_{1}=\frac{1}{\sqrt{1+R_{1}^{2}}}\left(\begin{array}{c}
1 \\
R_{1} e^{i \varphi_{1}}
\end{array}\right), \mathbf{i}_{2}=\frac{R_{2}}{\sqrt{1+R_{2}^{2}}}\left(\begin{array}{c}
1 \\
\frac{1}{R_{2}} e^{-i \varphi_{2}}
\end{array}\right) .
$$

whereas $R_{1}(\omega) \neq R_{2}(\omega)$ and $\varphi_{1}(\omega) \neq \varphi_{2}(\omega)$. The eigenstates are strongly depending on the actual value of the components of $\hat{T}$ and are simply elliptical, whereas no principal rotation direction is assignable. Linear as well as elliptical counter- and co-rotating states and combinations of them with no fixed phase relation can be found in general.

An example of such an structure is investigated in detail both numerically and experimentally in [23].

\section{SUMMARY}

A summarizing overview of possible structures and the corresponding basic forms of the T-matrices are shown in 
Table [1. Once the general form of the T-matrix is known all effects regarding the observable polarization phenomena can be fully deduced. Based on our investigations it is easy to provide an algorithm to determine the general form of the T-matrix for an unknown sample by measuring transmitted intensities with the help of linear polarizers only.

A possible approach can be as follows:

1. Use linearly polarized light and measure the orthog-

\begin{tabular}{||l|c|c|l||}
\hline \hline symmetry & examples & T-matrix & eigenstates \\
\hline$M_{x z}\left(M_{y z}\right)$ & & $T=\left(\begin{array}{ll}A & 0 \\
0 & D\end{array}\right)$ & linear \\
\hline $\begin{array}{l}C_{4, z}\left(C_{3, z}\right) \\
M_{x y}\left(C_{2, z},\right.\end{array}$ & & $T=\left(\begin{array}{cc}A & B \\
-B & A\end{array}\right)$ & circular \\
inversion \\
symmetry $)$
\end{tabular}

TABLE I: Overview of possible symmetries, typical metaatoms, the corresponding T-matrices and their eigenstates of the polarization. For every symmetry group only a single example is shown. Other possible symmetries resulting in the same type of T-matrices are given in brackets. Here $M_{i j}$ designates mirror symmetry with respect to the $i j$-plane and $C_{n}, i$ means n-fold rotational symmetry with respect to the $i$-axis.

onally polarized output while rotating the sample. If the output vanishes for every rotation angle the medium is polarization independent, i.e., a simple isotropic medium. If the output vanishes for some rotation angles and this angle is independent of the wavelength, the structure is simple anisotropic. If no such rotation angle can be found there is obviously no mirror plane parallel to the $z$-axis. 2 . If the transmitted intensity is independent of the rotation of the sample for both co- and cross-polarized light, the eigenstates are circular polarized and the structure is simple chiral.

3. If both aforementioned procedures do not provide a positive results, the structure is more complex and the measurements become more difficult too. To distinguish between the remaining possible forms it is necessary to measure the off-diagonal entries of the T-matrix simultaneously. If these off-diagonal elements are identical for a fixed wavelength and a fixed rotation angle independent of their particular choice, the structure is generalized anisotropic. If the off-diagonal elements are identical only for a fixed rotation angle but for every wavelengths the structure is generalized chiral. In all other cases we have $A \neq B \neq C \neq D$.

By using circular polarized light a similar scheme can be obtained however it would require circular analyzers as well.

\section{CONCLUSION}

Taking advantage of symmetry considerations we have analyzed the potential of various MMs to affect the polarization state of light upon transmission. By focusing the attention on any optical response that is directly accessible in an experiment, the properties of MMs may become so involved that the establishment of valid constitutive relation may be beyond what is possible for structures with an ever increasing complexity. We have explicitly shown that all MMs belong to one of five different classes; each being characterized by certain relations that connect the entries of the T-matrix and each class is able to support specific polarization phenomena. The sub-wavelength nature of $\mathrm{MMs}$ is the only requirement for these considerations. Moreover, the symmetry operations applied to the metaatoms have to be consistent with the symmetry of the lattice and it is required that the MM is sandwiched between identical media. Nonetheless, we have explicitly listed all relevant structures where a violation of this assumption causes deviations. To foster practical application of this classification we have finally provided a protocol useful to reveal the underlying symmetry of an unknown MM and its T-matrix from far-field measurements of the transmitted intensities only. Once it is identified, all the achievable optical properties that affect the state of polarization are fully disclosed.

\section{Acknowledgements}

We acknowledge financial support from the German Federal Ministry of Education and Research (Metamat and $\mathrm{PhoNa}$ ) and by the Thuringian State Government (MeMa).
[1] S. Linden, C. Enkrich, M. Wegener, J. Zhou, T. Koschny, C. M. Soukoulis, Science 306, 1351 (2004).
[2] C. Enkrich, M. Wegener, S. Linden, S. Burger, L. 
Zschiedrich, F. Schmidt, J. F. Zhou, Th. Koschny, and C. M. Soukoulis, Phys. Rev. Lett. 95, 203901 (2005).

[3] C. M. Soukoulis, S. Linden, and M. Wegener, Science 315, 47 (2007).

[4] W. J. Padilla, M. T. Aronsson, C. Highstrete, Mark Lee, A. J. Taylor, and R. D. Averitt, Phys. Rev. B 75, 041102(R) (2007).

[5] C. M. Bingham, H. Tao, X. Liu, R. D. Averitt, X. Zhang, and W. J. Padilla, Opt Exp. 16, 18565 (2008).

[6] M. Decker, S. Linden, and M. Wegener, Opt. Lett. 34, 1579 (2009).

[7] N. Liu, H. Liu, S. Zhu and H.Giessen, Nat. Phot. 3, 157 (2009).

[8] A.V. Rogacheva, V.A. Fedotov, A. S. Schwanecke, and N. I. Zheludev, Phys. Rev. Lett. 97, 177401 (2006).

[9] S. Zhang, Y.-S. Park, J. Li, X. Lu, W. Zhang, and X. Zhang, Phys. Rev. Lett. 102, 023901 (2009).

[10] E. Plum, J. Zhou, J. Dong, V. A. Fedotov, T. Koschny, C. M. Soukoulis, and N. I. Zheludev, Phys. Rev. B 79, 035407 (2009).

[11] J. Zhou, J. Dong, B. Wang, T. Koschny, M. Kafesaki, and C. M. Soukoulis, Phys. Rev. B 79, 121104(R) (2009).

[12] B. Wang, J. Zhou, T. Koschny, M. Kafesaki, and C. M. Soukoulis, J. Opt. A 11, 114003 (2009).

[13] C. Rockstuhl, C. Menzel, T. Paul, and F. Lederer, Phys. Rev. B 79, 035321 (2009).

[14] Yuqian Ye and Sailing He, Appl. Phys. Lett. 96, 203501 (2010).

[15] B. Bai, Y. Svirko, J. Turunen, and T. Vallius, Phys. Rev. A 76, 023811 (2007).

[16] C. Menzel, C. Rockstuhl, T. Paul, and F. Lederer, Appl. Phys. Lett. 93, 233106 (2008).

[17] K. Jefimovs, N. Saito, Yu. Ino, T. Vallius, P. Vahimaa, J. Turunen, R. Shimano, M. Kauranen, Yu. Svirko, M. Kuwata-Gonokami, Microelec. Eng. 78, 448 (2005).

[18] J. K. Gansel, M. Thiel, M. S. Rill, M. Decker, K. Bade, V. Saile, G. v. Freymann, S. Linden, and M. Wegener, Science 325, 1513 (2009).

[19] V. A. Fedotov, P. L. Mladyonov, S. L. Prosvirnin, A.V. Rogacheva, Y. Chen, and N. I. Zheludev, Phys. Rev. Lett. 97, 167401 (2006).

[20] V. A. Fedotov, A. S. Schwanecke, N. I. Zheludev, V. V. Khardikov, and S. L. Prosvirnin, Nano Lett. 7, 1996 (2007).

[21] A. S. Schwanecke, V. A. Fedotov, V. V. Khardikov, S. L. Prosvirnin, Y. Chen, and N. I. Zheludev, Nano Lett. 8, 2940 (2008).

[22] R. Singh, E. Plum, C. Menzel, C. Rockstuhl, A. K. Azad, R. A. Cheville, F. Lederer, W. Zhang, and N. I. Zheludev, Phys. Rev. B 80, 153104 (2009).

[23] C. Menzel, C. Helgert, C. Rockstuhl,1 E.-B. Kley, A. Tünnermann, T. Pertsch, and F. Lederer, Phys. Rev. Lett. 104, 253902 (2010).

[24] C. Menzel, T. Paul, C. Rockstuhl, T. Pertsch, S. Tretyakov, and F. Lederer, Phys. Rev. B 81, 035320 (2010).
[25] Willie J. Padilla, Opt. Exp. 15, 1639 (2007).

[26] L.R. Arnaut, J. Electromagn. Waves Appl. 11, 1459 (1997).

[27] A. Serdyukov, I. Semchenko, S. Tretyakov, and A. Sihvola, Electromagnetics of Bi-Anisotropic Materials Theory and Applications, Gordon and Breach Science Publ. (2001).

[28] E. Plum, V. A. Fedotov, A. S. Schwanecke, N. I. Zheludev, and Y. Chen, Appl. Phys. Lett. 90, 223113 (2007).

[29] R. C. Jones, J. Opt. Soc. Am. 31, 488 (1941).

[30] A. Drezet, C. Genet, J.-Y. Laluet, and T. W. Ebbesen, Opt. Exp. 16, 12559 (2008).

[31] Shih-Yau Lu and Russell A. Chipman, J. Opt. Soc. Am. A 13, 1106 (1996).

[32] R. J. Potton, Rep. Prog. Phys. 67, 717 (2004).

[33] Sudha and A. V. Gopala Rao, J. Opt. Soc. Am. A 18, 3130 (2001).

[34] S. N. Savenkov, O. I. Sydoruk, and R. S. Muttiah, Appl. Opt. 46, 6700 (2007).

[35] O. Sydoruk, and S. N. Savenkov, J. Opt. 12, 035702 (2010).

[36] Shih-Yau Lu and Russell A. Chipman, J. Opt. Soc. Am. A 11, 766 (1994).

[37] S. I. Maslovski, D. K. Morits and S. A. Tretyakov, J. Opt. A 11, 074004 (2009).

[38] S. N. Volkov, K. Dolgaleva, R. W. Boyd, K. Jefimovs, J. Turunen, Y. Svirko, B. K. Canfield, and M. Kauranen, Phys. Rev. A 79, 043819 (2009).

[39] S. Zhang, W. Fan, K. J. Malloy, S.R. Brueck, N. C. Panoiu, and R. M. Osgood, Opt. Exp. 13, 4922 (2005).

[40] M. Kafesaki, I. Tsiapa, N. Katsarakis, Th. Koschny, C. M. Soukoulis, and E. N. Economou, Phys. Rev. B 75, 235114 (2007).

[41] V. M. Shalaev, Nat. Phot. 1, 41 (2007).

[42] M. Decker, M. W. Klein, M. Wegener, and S. Linden, Opt. Lett. 32, 856 (2007).

[43] Eric Plum, Chirality and Metamaterials - PhD-thesis (2010).

[44] E. Plum, V. A. Fedotov, and N. I. Zheludev, Appl. Phys. Lett. 94, 131901 (2009).

[45] S. V. Zhukovsky, A. V. Novitsky, and V. M. Galynsky, Opt. Lett. 34, 1988 (2009).

[46] J. Petschulat, A. Chipouline, A. Tünnermann, T. Pertsch, C. Menzel, C. Rockstuhl, T. Paul, and F. Lederer, arXiv:0909.0778v2 (2010)

[47] J. C. W. Lee, and C.T. Chan, Opt. Expr. 13, 8083 (2005).

[48] J. K. Gansel, M. Wegener, S. Burger, and S. Linden, Opt. Expr. 18, 1059 (2010).

[49] M. G. Silveirinha, IEEE Trans. Anten. and Prop. 56, 390 (2008).

[50] I. Hodgkinson, Q. H. Wu, B. Knight, A. Lakhtakia, and K. Robbie, Appl. Opt. 39, 642 (2000).

[51] A. Lakhtakia, V. C. Venugopal, M. W. McCall, Opt. Comm. 177, 57 (2000). 